Advances in Dynamical Systems and Applications

ISSN 0973-5321, Volume 14, Number 1, pp. 1-27 (2019)

https://dx.doi.org/10.37622/ADSA/14.1.2019.1-27

\title{
Nabla Time Scales Iyengar-Type Inequalities
}

\author{
George A. Anastassiou \\ University of Memphis \\ Department of Mathematical Sciences \\ Memphis, TN 38152, U.S.A.
}

\begin{abstract}
Here we present the necessary background on nabla time scales approach. Then we give general related time scales nabla Iyengar type inequalities for all basic norms. We finish with applications to specific time scales like $\mathbb{R}, \mathbb{Z}$ and $q^{\bar{Z}}, q>1$.
\end{abstract}

AMS Subject Classifications: 26D15, 39A12, 93C70.

Keywords: Iyengar inequality, time scale, nabla calculus.

\section{Introduction}

We are motivated by the following famous Iyengar inequality (1938), [8].

Theorem 1.1. Let $f$ be a differentiable function on $[a, b]$ and $\left|f^{\prime}(x)\right| \leq M$. Then

$$
\left|\int_{a}^{b} f(x) d x-\frac{1}{2}(b-a)(f(a)+f(b))\right| \leq \frac{M(b-a)^{2}}{4}-\frac{(f(b)-f(a))^{2}}{4 M} .
$$

We present generalized analogs of (1.1) to time scales in the nabla sense. Motivation comes also from [1-3].

\section{Background}

Here we follow $[5-7,10]$. Let $\mathbb{T}$ be a time scale (a closed subset of $\mathbb{R})([8]),[a, b]$ be the closed and bounded interval in $\mathbb{T}$, i.e. $[a, b]:=\{t \in \mathbb{T}: a \leq t \leq b\}$ and $a, b \in \mathbb{T}$.

Received January 14, 2019; Accepted July 1, 2019

Communicated by Martin Bohner 
Clearly, a time scale $\mathbb{T}$ may or may not be connected. Therefore we have the concept of forward and backward jump operators as follows. Define $\sigma, \rho: \mathbb{T} \longmapsto \mathbb{T}$ by

$$
\sigma(t)=\inf \{s \in \mathbb{T}: s>t\} \text { and } \rho(t)=\sup \{s \in \mathbb{T}: s<t\},
$$

$(\inf \emptyset:=\sup \mathbb{T}, \sup \emptyset:=\inf \mathbb{T})$.

If $\sigma(t)=t, \sigma(t)>t, \rho(t)=t, \rho(t)<t$, then $t \in \mathbb{T}$ is called right-dense, rightscattered, left-dense, left-scattered, respectively. The set $\mathbb{T}_{k}$ which is derived from $\mathbb{T}$ is as follows: if $\mathbb{T}$ has a right-scattered minimum $m$, then $\mathbb{T}_{k}=\mathbb{T}-\{m\}$, otherwise $\mathbb{T}_{k}=\mathbb{T}$. We also define the backwards graininess function $\nu: \mathbb{T}_{k} \longmapsto[0, \infty)$ as $\nu(t)=t-\rho(t)$. If $f: \mathbb{T} \longmapsto \mathbb{R}$ is a function, we define the function $f^{\rho}: \mathbb{T}_{k} \longmapsto \mathbb{R}$ by $f^{\rho}(t)=f(\rho(t))$ for all $t \in \mathbb{T}_{k}$ and $\sigma^{0}(t)=\rho^{0}(t)=t ; \mathbb{T}_{k^{n+1}}:=\left(\mathbb{T}_{k^{n}}\right)_{k}$.

Definition 2.1. If $f: \mathbb{T} \longmapsto \mathbb{R}$ is a function and $t \in \mathbb{T}_{k}$, then we define the nabla derivative of $f$ at a point $t$ to be the number $f^{\nabla}(t)$ (provided it exists) with the property that, for each $\varepsilon>0$, there is a neighborhood of $U$ of $t$ such that

$$
\left|[f(\rho(t))-f(s)]-f^{\nabla}(t)[\rho(t)-s]\right| \leq \varepsilon|\rho(t)-s|
$$

for all $s \in U$.

Note that in the case $\mathbb{T}=\mathbb{R}$, then $f^{\nabla}(t)=f^{\prime}(t)$, and if $\mathbb{T}=\mathbb{Z}$, then $f^{\nabla}(t)=$ $\nabla f(t)=f(t)-f(t-1)$.

Definition 2.2. A function $F: \mathbb{T} \rightarrow \mathbb{R}$ we call a nabla-antiderivative of $f: \mathbb{T} \rightarrow \mathbb{R}$ provided that $F^{\nabla}(t)=f(t)$ for all $t \in \mathbb{T}_{k}$. We then define the Cauchy $\nabla$-integral from $a$ to $t$ of $f$ by

$$
\int_{a}^{t} f(s) \nabla s=F(t)-F(a), \text { for all } t \in \mathbb{T} .
$$

Note that in the case $\mathbb{T}=\mathbb{R}$ we have

$$
\int_{a}^{b} f(t) \nabla t=\int_{a}^{b} f(t) d t
$$

and in the case $\mathbb{T}=\mathbb{Z}$ we have

$$
\int_{a}^{b} f(t) \nabla t=\sum_{k=a+1}^{b} f(k),
$$

where $a, b \in \mathbb{T}$ with $a \leq b$.

Definition 2.3. A function $f: \mathbb{T} \rightarrow \mathbb{R}$ is left-dense continuous (or ld-continuous) provided that it is continuous at left-dense points in $\mathbb{T}$ and its right-sided limits exist at right-dense points of $\mathbb{T}$.

If $\mathbb{T}=\mathbb{R}$, then $f$ is ld-continuous iff $f$ is continuous. If $\mathbb{T}=\mathbb{Z}$, then any function is ld-continuous. 
Theorem 2.4. Let $\mathbb{T}$ be a time scale, $f: \mathbb{T} \rightarrow \mathbb{R}$, and $t \in \mathbb{T}_{k}$. The following holds:

1. If $f$ is nabla differentiable at $t$, then $f$ is continuous at $t$.

2. If $f$ is continuous at $t$ and $t$ is left-scattered, then $f$ is nabla differentiable at $t$ and

$$
f^{\nabla}(t)=\frac{f(t)-f(\rho(t))}{t-\rho(t)} .
$$

3. If t is left-dense, then $f$ is nabla differentiable at $t$ if and only if the limit

$$
\lim _{s \rightarrow t} \frac{f(t)-f(s)}{t-s}
$$

exists as a finite number. In this case,

$$
f^{\nabla}(t)=\lim _{s \rightarrow t} \frac{f(t)-f(s)}{t-s} .
$$

4. If $f$ is nabla differentiable at $t$, then $f(\rho(t))=f(t)-\nu(t) f^{\nabla}(t)$.

For any time scale $\mathbb{T}$, when $f$ is a constant, then $f^{\nabla}=0$; if $f(t)=k t$ for some constant $k$, then $f^{\nabla}=k$.

Theorem 2.5. Suppose $f, g: \mathbb{T} \rightarrow \mathbb{R}$ are nabla differentiable at $t \in \mathbb{T}_{k}$. Then,

1. the sum $f+g: \mathbb{T} \rightarrow \mathbb{R}$ is nabla differentiable at $t$ and

$$
(f+g)^{\nabla}(t)=f^{\nabla}(t)+g^{\nabla}(t)
$$

2. for any constant $\alpha, \alpha f: \mathbb{T} \rightarrow \mathbb{R}$ is nabla differentiable at $t$ and

$$
(\alpha f)^{\nabla}(t)=\alpha f^{\nabla}(t)
$$

3. the product $f g: \mathbb{T} \rightarrow \mathbb{R}$ is nabla differentiable at $t$ and

$$
(f g)^{\nabla}(t)=f^{\nabla}(t) g(t)+f^{\rho}(t) g^{\nabla}(t)=f^{\nabla}(t) g^{\rho}(t)+f(t) g^{\nabla}(t) .
$$

Some results concerning ld-continuity are useful:

Theorem 2.6. Let $\mathbb{T}$ be a time scale, $f: \mathbb{T} \rightarrow \mathbb{R}$.

1. If $f$ is continuous, then $f$ is ld-continuous.

2. The backward jump operator $\rho$ is ld-continuous.

3. If $f$ is ld-continuous, then $f^{\rho}$ is also ld-continuous. 
4. If $\mathbb{T}=\mathbb{R}$, then $f$ is continuous if and only if $f$ is ld-continuous.

5. If $\mathbb{T}=\mathbb{Z}$, then $f$ is ld-continuous.

Theorem 2.7. Every ld-continuous function has a nabla antiderivative. In particular, if $a \in \mathbb{T}$, then the function $F$ defined by

$$
F(t)=\int_{a}^{t} f(\tau) \nabla \tau, \quad t \in \mathbb{T},
$$

is a nabla antiderivative of $f$.

The set of all ld-continuous functions $f: \mathbb{T} \rightarrow \mathbb{R}$ is denoted by $C_{l d}(\mathbb{T}, \mathbb{R})$, and the set of all nabla differentiable functions with ld-continuous derivative by $C_{l d}^{1}(\mathbb{T}, \mathbb{R})$.

Theorem 2.8. If $f \in C_{l d}(\mathbb{T}, \mathbb{R})$ and $t \in \mathbb{T}_{k}$, then

$$
\int_{\rho(t)}^{t} f(\tau) \nabla \tau=\nu(t) f(t) .
$$

Theorem 2.9. If $a, b, c \in \mathbb{T}, a \leq c \leq b, \alpha \in \mathbb{R}$, and $f, g \in C_{l d}(\mathbb{T}, \mathbb{R})$, then:

$$
\begin{aligned}
& \text { 1. } \int_{a}^{b}(f(t)+g(t)) \nabla t=\int_{a}^{b} f(t) \nabla t+\int_{a}^{b} g(t) \nabla t ; \\
& \text { 2. } \int_{a}^{b} \alpha f(t) \nabla t=\alpha \int_{a}^{b} f(t) \nabla t ; \\
& \text { 3. } \int_{a}^{b} f(t) \nabla t=-\int_{b}^{a} f(t) \nabla t ; \\
& \text { 4. } \int_{a}^{a} f(t) \nabla t=0 ; \\
& \text { 5. } \int_{a}^{b} f(t) \nabla t=\int_{a}^{c} f(t) \nabla t+\int_{c}^{b} f(t) \nabla t ; \\
& \text { 6. If } f(t)>0 \text { for all } a<t \leq b, \text { then } \int_{a}^{b} f(t) \nabla t>0 ; \\
& \text { 7. } \int_{a}^{b} f^{\rho}(t) g^{\nabla}(t) \nabla t=[(f g)(t)]_{t=a}^{t=b}-\int_{a}^{b} f^{\nabla}(t) g(t) \nabla t \text {; } \\
& \text { 8. } \int_{a}^{b} f(t) g^{\nabla}(t) \nabla t=[(f g)(t)]_{t=a}^{t=b}-\int_{a}^{b} f^{\nabla}(t) g^{\rho}(t) \nabla t \text {; }
\end{aligned}
$$


9. If $f(t) \geq 0, a \leq t \leq b$, then $\int_{a}^{b} f(t) \nabla t \geq 0$;

10. If $f(t) \geq 0, a \leq c \leq b$, then $\int_{a}^{b} f(t) \nabla t \geq \int_{a}^{c} f(t) \nabla t$;

11. If $f$ and $f^{\nabla}$ are jointly continuous in $(t, s)$, then

$$
\begin{aligned}
& \left(\int_{a}^{t} f(t, s) \nabla s\right)^{\nabla}=f(\rho(t), t)+\int_{a}^{t} f^{\nabla}(t, s) \nabla s \\
& \left(\int_{t}^{b} f(t, s) \nabla s\right)^{\nabla}=-f(\rho(t), t)+\int_{t}^{b} f^{\nabla}(t, s) \nabla s
\end{aligned}
$$

12. If $f(t) \geq g(t)$, then $\int_{a}^{b} f(t) \nabla t \geq \int_{a}^{b} g(t) \nabla t$;

13. $\left|\int_{a}^{b} f(t) \nabla t\right| \leq \int_{a}^{b}|f(t)| \nabla t$

14. $\int_{a}^{b} 1 \nabla t=b-a$.

Similarly we define higher order nabla derivatives on $\mathbb{T}_{k^{n+1}}$ by

$$
f^{\nabla^{n+1}}:=\left(f^{\nabla^{n}}\right)^{\nabla}, \quad n \in \mathbb{N} .
$$

If $\mathbb{T}=\mathbb{R}$, then $f^{\nabla^{n+1}}=f^{(n+1)}$, and if $\mathbb{T}=\mathbb{Z}$, then $f^{\nabla^{n+1}}(t)=\nabla^{n+1} f(t)=$ $\sum_{m=0}^{n+1}(-1)^{m}\left(\begin{array}{c}n+1 \\ m\end{array}\right) f(t-m)$.

Let $\widehat{h}_{k}: \mathbb{T}^{2} \rightarrow \mathbb{R}, k \in \mathbb{N}_{0}=\mathbb{N} \cup\{0\}$, defined recursively as follows:

$$
\widehat{h}_{0}(t, s)=1, \text { all } s, t \in \mathbb{T},
$$

and, given $\widehat{h}_{k}$ for $k \in \mathbb{N}_{0}$, the function $\widehat{h}_{k+1}$ is

$$
\widehat{h}_{k+1}(t, s)=\int_{s}^{t} \widehat{h}_{k}(\tau, s) \nabla \tau, \text { for all } s, t \in \mathbb{T} \text {. }
$$

Note that $\widehat{h}_{k}$ are all well defined, since each is ld-continuous in $t$.

If we let $\widehat{h}_{k}^{\nabla}(t, s)$ denote for each fixed $s$ the nabla derivative of $\widehat{h}_{k}(t, s)$ with respect to $t$, then

$$
\widehat{h}_{k}^{\nabla}(t, s)=\widehat{h}_{k-1}(t, s), \quad \text { for } k \in \mathbb{N}, t \in \mathbb{T}_{k} .
$$

Notice that $\widehat{h}_{1}(t, s)=t-s$, for all $s, t \in \mathbb{T}$.

By [2] we have that $\widehat{h}_{k}(t, s) \geq 0$, for any $t, s \in \mathbb{T}$, when $k$ is even. 
Example 2.10. 1. If $\mathbb{T}=\mathbb{R}$, then $\rho(t)=t, t \in \mathbb{R}$, so that $\widehat{h}_{k}(t, s)=\frac{(t-s)^{k}}{k !}$ for all $s, t \in \mathbb{R}, k \in \mathbb{N}_{0}$.

2. If $\mathbb{T}=\mathbb{Z}$, then $\rho(t)=t-1, t \in \mathbb{Z}$, and $\widehat{h}_{k}(t, s)=\frac{(t-s)^{\bar{k}}}{k !}$, for all $s, t \in \mathbb{Z}$, $k \in \mathbb{N}_{0}$, where $t^{\bar{k}}:=t(t+1) \ldots(t+k-1), k \in \mathbb{N} ; t^{\overline{0}}:=1$.

Definition 2.11. The set $C_{l d}^{n}(\mathbb{T}, \mathbb{R})=C_{l d}^{n}(\mathbb{T}), n \in \mathbb{N}$, denotes the set of all $n$ times continuously nabla differentiable functions from $\mathbb{T}$ into $\mathbb{R}$, i.e. all $f, f^{\nabla}, f^{\nabla^{2}}, \ldots, f^{\nabla^{n}} \in$ $C_{l d}(\mathbb{T}, \mathbb{R})$.

This definition requires $\mathbb{T}_{k}=\mathbb{T}$.

We need the following result.

Theorem 2.12 (Nabla Taylor Formula, see [4]). Suppose $f$ is $n$ times nabla differentiable on $\mathbb{T}_{k^{n}}, n \in \mathbb{N}$. Let $a \in \mathbb{T}_{k^{n-1}}, t \in \mathbb{T}$. Then

$$
f(t)=\sum_{k=0}^{n-1} \widehat{h}_{k}(t, a) f^{\nabla^{k}}(a)+\int_{a}^{t} \widehat{h}_{n-1}(t, \rho(\tau)) f^{\nabla^{n}}(\tau) \nabla \tau .
$$

If $f \in C_{l d}^{n}(\mathbb{T}, \mathbb{R})$, then nabla Taylor formula is true for all $t, a \in \mathbb{T}$.

Corollary 2.13 (to Theorem 2.12). Assume $f \in C_{l d}^{n}(\mathbb{T}), n \in \mathbb{N}$, and $s, t \in \mathbb{T}$. Let $m \in \mathbb{N}$ with $m<n$. Then

$$
f^{\nabla^{m}}(t)=\sum_{k=0}^{n-m-1} f^{\nabla^{k+m}}(s) \widehat{h}_{k}(t, s)+\int_{s}^{t} \widehat{h}_{n-m-1}(t, \rho(\tau)) f^{\nabla^{n}}(\tau) \nabla \tau .
$$

Proof. Use Theorem 2.12 with $n$ and $f$ substituted by $n-m$ and $f^{\nabla^{m}}$, respectively.

Define $[a, b]_{k}=[a, b]$ if $a$ is right-dense, and $[a, b]_{k}=[\sigma(a), b]$ if $a$ is rightscattered.

Proposition 2.14 (See [10]). Suppose $a, b \in \mathbb{T}, a<b$, and $f \in C_{l d}([a, b], \mathbb{R})$ is such that $f \geq 0$ on $[a, b]$. If $\int_{a}^{b} f(t) \nabla t=0$, then $f=0$ on $[a, b]_{k}$.

Theorem 2.15 (Nabla Hölder Inequality, see [2]). Let $a, b \in \mathbb{T}, a \leq b$. For $f, g \in$ $C_{l d}([a, b])$ we have

$$
\int_{a}^{b}|f(t)||g(t)| \nabla t \leq\left(\int_{a}^{b}|f(t)|^{p} \nabla t\right)^{\frac{1}{p}} \cdot\left(\int_{a}^{b}|g(t)|^{q} \nabla t\right)^{\frac{1}{q}},
$$

where $p, q>1: \frac{1}{p}+\frac{1}{q}=1$. 
Next define $\widehat{g}_{0}(t, s) \equiv 1$,

$$
\widehat{g}_{n+1}(t, s)=\int_{s}^{t} \widehat{g}_{n}(\rho(\tau), s) \nabla \tau, \quad n \in \mathbb{N}, s, t \in \mathbb{T} .
$$

Notice that $\widehat{g}_{n+1}^{\nabla}(t, s)=\widehat{g}_{n}(\rho(t), s), t \in \mathbb{T}_{k} ; \widehat{g}_{1}(t, s)=t-s$, for all $s, t \in \mathbb{T}$.

If $\mathbb{T}$ has a left-scattered maximum $M$, define $\mathbb{T}^{k}:=\mathbb{T}-\{M\}$; otherwise, set $\mathbb{T}^{k}=\mathbb{T}$. Similarly define $\mathbb{T}^{k^{n+1}}:=\left(\mathbb{T}^{k^{n}}\right)^{k}$. Notice $\mathbb{T}_{k^{n+1}} \subset \mathbb{T}_{k}$ and $\mathbb{T}^{k^{n+1}} \subset \mathbb{T}^{k}$.

Theorem 2.16 (See [4]). Let $t \in \mathbb{T}_{k} \cap \mathbb{T}^{k}, s \in \mathbb{T}^{k^{n}}$, and $n \geq 0$. Then

$$
\widehat{h}_{n}(t, s)=(-1)^{n} \widehat{g}_{n}(s, t) \text {. }
$$

Remark 2.17. Let the time scale $\mathbb{T}$ be such that $\mathbb{T}^{k}=\mathbb{T}_{k}=\mathbb{T}$. Clearly both $\widehat{h}_{n}, \widehat{g}_{n}$ are nabla differentiable in their first variables, therefore both are continuous in their first variables.

Using now Theorem 2.16 we get that also both $\widehat{h}_{n}, \widehat{g}_{n}$ are continuous in their second variables.

Consequently $\widehat{h}_{n}(t, s)$ is ld-continuous in each variable and thus $\widehat{h}_{n}(t, \rho(s))$ is ldcontinuous in $s$.

Notice also in general that if $t \geq s$ then $\widehat{h}_{1}(t, s) \geq 0, \widehat{h}_{2}(t, s) \geq 0, \ldots, \widehat{h}_{n-1}(t, s) \geq$ 0 . So that $\widehat{h}_{n-1}(t, \rho(\tau)) \geq 0$ for all $s \leq \tau \leq t$.

Also in general it holds

$$
\widehat{h}_{k}(t, s) \leq(t-s)^{k}, \forall t \geq s, k \in \mathbb{N}_{0},
$$

and easily we get:

$$
\left|\widehat{h}_{k}(t, s)\right| \leq|t-s|^{k}, \forall t, s \in \mathbb{T}, k \in \mathbb{N}_{0}
$$

We need the following result.

Theorem 2.18 (Nabla Chain Rule, see [6]). Let $f: \mathbb{R} \rightarrow \mathbb{R}$ be continuously differentiable and suppose that $g: \mathbb{T} \rightarrow \mathbb{R}$ is nabla differentiable on $\mathbb{T}$. Then $f \circ g: \mathbb{T} \rightarrow \mathbb{R}$ is nabla differentiable on $\mathbb{T}$ and the formula

$$
(f \circ g)^{\nabla}(t)=\left\{\int_{0}^{1} f^{\prime}\left(g(t)+h \nu(t) g^{\nabla}(t)\right) d h\right\} g^{\nabla}(t)
$$

holds.

We formulate the following assumption.

Assumption 2.19. Let the time scale $\mathbb{T}$ be such that $\mathbb{T}^{k}=\mathbb{T}_{k}=\mathbb{T}$. 
Remark 2.20. Assume that $\rho$ is a continuous function, $\mathbb{T}_{k}=\mathbb{T}, \widehat{h}_{n-1}(t, s)$ and $\widehat{h}_{n-2}(t, s)$ are jointly continuous in $(t, s) \in \mathbb{T}^{2} ; p>1$. Clearly $\widehat{h}_{n-1}^{\nabla}(t, s)=\widehat{h}_{n-2}(t, s)$ in $t \in \mathbb{T}$. Also $\widehat{h}_{n-1}(t, \rho(s)), \widehat{h}_{n-2}(t, \rho(s))$ are jointly continuous in $(t, s) \in \mathbb{T}^{2}$.

By Theorem 2.18 we have that $\left(\left(\widehat{h}_{n-1}(t, \rho(\tau))\right)^{p}\right)^{\nabla}$ exists in $t \in \mathbb{T}$, where $\tau$ is fixed in $\mathbb{T}$, and

$$
\begin{gathered}
\left(\left(\widehat{h}_{n-1}(t, \rho(\tau))\right)^{p}\right)^{\nabla}= \\
p\left\{\int_{0}^{1}\left(\widehat{h}_{n-1}(t, \rho(\tau))+h \nu(t) \widehat{h}_{n-2}(t, \rho(\tau))\right)^{p-1} d h\right\} \widehat{h}_{n-2}(t, \rho(\tau)) .
\end{gathered}
$$

By bounded convergence theorem we obtain that $\left(\left(\widehat{h}_{n-1}(t, \rho(\tau))\right)^{p}\right)^{\nabla}$ is jointly continuous in $(t, \tau)$, and of course $\left(\widehat{h}_{n-1}(t, \rho(\tau))\right)^{p}$ is jointly continuous in $(t, \tau)$.

Therefore by Theorem 2.9 (11), we derive for

$$
u(t)=\int_{a}^{t} \widehat{h}_{n-1}(t, \rho(\tau))^{p} \nabla \tau
$$

$(t \in[a, b] \subset \mathbb{T})$, that

$$
u^{\nabla}(t)=\int_{a}^{b}\left(\widehat{h}_{n-1}(t, \rho(\tau))^{p}\right)^{\nabla} \nabla \tau+\left(\widehat{h}_{n-1}(\rho(t), \rho(t))\right)^{p}
$$

I.e.

$$
u^{\nabla}(t)=\int_{a}^{t}\left(\widehat{h}_{n-1}(t, \rho(\tau))^{p}\right)^{\nabla} \nabla \tau .
$$

That is $u(t)$ is nabla differentiable, hence continuous and therefore ld-continuous on $[a, b] \subset \mathbb{T}$.

We formulate the next assumptions.

Assumption 2.21. We suppose that $\rho$ is a continuous function and

$$
\widehat{h}_{n-1}(t, s), \quad \widehat{h}_{n-2}(t, s)
$$

are jointly continuous in $(t, s) \in \mathbb{T}^{2}$.

Assumption 2.22. We suppose that $\rho$ is a continuous function and

$$
\widehat{h}_{n-m-1}(t, s), \quad \widehat{h}_{n-m-2}(t, s)
$$

are jointly continuous in $(t, s) \in \mathbb{T}^{2}$. 


\section{Main Results}

Next we present nabla Iyengar type inequalities on time scales for all norms $\|\cdot\|_{p}, 1 \leq$ $p \leq \infty$. We give the following result.

Theorem 3.1. Let $f \in C_{l d}^{n}(\mathbb{T}), n \in \mathbb{N}$ is odd, $a, b \in \mathbb{T} ; a \leq b$. Here $\rho$ is continuous and $\widehat{h}_{n-1}(t, s)$ is jointly continuous. Also assume that $\mathbb{T}_{k}=\mathbb{T}$. Then

1)

$$
\begin{gathered}
\left|\int_{a}^{b} f(t) \nabla t-\sum_{k=0}^{n-1}\left(f^{\nabla^{k}}(a) \widehat{h}_{k+1}(x, a)-f^{\nabla^{k}}(b) \widehat{h}_{k+1}(x, b)\right)\right| \leq\left\|f^{\nabla^{n}}\right\|_{\infty,[a, b] \cap \mathbb{T}} \\
{\left[\left(\int_{a}^{x}\left(\int_{a}^{t} \widehat{h}_{n-1}(t, \rho(\tau)) \nabla \tau\right) \nabla t\right)+\left(\int_{x}^{b}\left(\int_{t}^{b} \widehat{h}_{n-1}(t, \rho(\tau)) \nabla \tau\right) \nabla t\right)\right],}
\end{gathered}
$$

$\forall x \in[a, b] \cap \mathbb{T}$,

2) assuming $f^{\nabla^{k}}(a)=f^{\nabla^{k}}(b)=0, k=0,1, \ldots, n-1$, we get from (3.1) that

$$
\begin{gathered}
\left|\int_{a}^{b} f(t) \nabla t\right| \leq\left\|f^{\nabla^{n}}\right\|_{\infty,[a, b] \cap \mathbb{T}} \\
{\left[\left(\int_{a}^{x}\left(\int_{a}^{t} \widehat{h}_{n-1}(t, \rho(\tau)) \nabla \tau\right) \nabla t\right)+\left(\int_{x}^{b}\left(\int_{t}^{b} \widehat{h}_{n-1}(t, \rho(\tau)) \nabla \tau\right) \nabla t\right)\right],}
\end{gathered}
$$

$\forall x \in[a, b] \cap \mathbb{T}$,

$22_{1}$ when $x=a$ we get from (3.2) that

$$
\left|\int_{a}^{b} f(t) \nabla t\right| \leq\left\|f^{\nabla^{n}}\right\|_{\infty,[a, b] \cap \mathbb{T}}\left(\int_{a}^{b}\left(\int_{t}^{b} \widehat{h}_{n-1}(t, \rho(\tau)) \nabla \tau\right) \nabla t\right),
$$

$22_{2}$ ) when $x=b$ we get from (3.2) that

$$
\left|\int_{a}^{b} f(t) \nabla t\right| \leq\left\|f^{\nabla^{n}}\right\|_{\infty,[a, b] \cap \mathbb{T}}\left(\int_{a}^{b}\left(\int_{a}^{t} \widehat{h}_{n-1}(t, \rho(\tau)) \nabla \tau\right) \nabla t\right),
$$

$\left.2_{3}\right)$ by (3.3) and (3.4) we get

$$
\begin{aligned}
& \left|\int_{a}^{b} f(t) \nabla t\right| \leq\left\|f^{\nabla^{n}}\right\|_{\infty,[a, b] \cap \mathbb{T}} \times \\
& \min \left\{\int_{a}^{b}\left(\int_{t}^{b} \widehat{h}_{n-1}(t, \rho(\tau)) \nabla \tau\right) \nabla t, \int_{a}^{b}\left(\int_{a}^{t} \widehat{h}_{n-1}(t, \rho(\tau)) \nabla \tau\right) \nabla t\right\},
\end{aligned}
$$

and 
3) assuming $f^{\nabla^{k}}(a)=f^{\nabla^{k}}(b)=0, k=1, \ldots, n-1$, by (3.1) we have

$$
\begin{aligned}
& \left|\int_{a}^{b} f(t) \nabla t-[f(a)(x-a)+f(b)(b-x)]\right| \leq\left\|f^{\nabla^{n}}\right\|_{\infty,[a, b] \cap \mathbb{T}} \times \\
& \quad\left[\int_{a}^{x}\left(\int_{a}^{t} \widehat{h}_{n-1}(t, \rho(\tau)) \nabla \tau\right) \nabla \Delta t+\int_{x}^{b}\left(\int_{t}^{b} \widehat{h}_{n-1}(t, \rho(\tau)) \nabla \tau\right) \nabla t\right],
\end{aligned}
$$

$\forall x \in[a, b] \cap \mathbb{T}$.

Proof. By [7, p. 23], we have that $\left\|f^{\nabla^{n}}\right\|_{\infty,[a, b] \cap \mathbb{T}}<\infty$. By Theorem 2.12 we have

$$
f(t)-\sum_{k=0}^{n-1} f^{\nabla^{k}}(a) \widehat{h}_{k}(t, a)=\int_{a}^{t} \widehat{h}_{n-1}(t, \rho(\tau)) f^{\nabla^{n}}(\tau) \nabla \tau,
$$

and

$$
f(t)-\sum_{k=0}^{n-1} f^{\nabla^{k}}(b) \widehat{h}_{k}(t, b)=\int_{b}^{t} \widehat{h}_{n-1}(t, \rho(\tau)) f^{\nabla^{n}}(\tau) \nabla \tau,
$$

$\forall t \in[a, b] \cap \mathbb{T}$.

Then we get

$$
\left|f(t)-\sum_{k=0}^{n-1} f^{\nabla^{k}}(a) \widehat{h}_{k}(t, a)\right| \stackrel{(3.7)}{\leq}\left\|f^{\nabla^{n}}\right\|_{\infty,[a, b] \cap \mathbb{T}} \int_{a}^{t} \widehat{h}_{n-1}(t, \rho(\tau)) \nabla \tau,
$$

and

$$
\begin{gathered}
\left|f(t)-\sum_{k=0}^{n-1} f^{\nabla^{k}}(b) \widehat{h}_{k}(t, b)\right| \stackrel{(3.8)}{=}\left|\int_{t}^{b} \widehat{h}_{n-1}(t, \rho(\tau)) f^{\nabla^{n}}(\tau) \nabla \tau\right| \\
\leq\left(\int_{t}^{b} \widehat{h}_{n-1}(t, \rho(\tau)) \nabla \tau\right)\left\|f^{\nabla^{n}}\right\|_{\infty,[a, b] \cap \mathbb{T}} .
\end{gathered}
$$

Therefore it holds (by (3.9), (3.10))

$$
\begin{gathered}
-\left\|f^{\nabla^{n}}\right\|_{\infty,[a, b] \cap \mathbb{T}} \int_{a}^{t} \widehat{h}_{n-1}(t, \rho(\tau)) \nabla \tau \leq f(t)-\sum_{k=0}^{n-1} f^{\nabla^{k}}(a) \widehat{h}_{k}(t, a) \\
\leq\left\|f^{\nabla^{n}}\right\|_{\infty,[a, b] \cap \mathbb{T}} \int_{a}^{t} \widehat{h}_{n-1}(t, \rho(\tau)) \nabla \tau
\end{gathered}
$$

and

$$
-\left\|f^{\nabla^{n}}\right\|_{\infty,[a, b] \cap \mathbb{T}}\left(\int_{t}^{b} \widehat{h}_{n-1}(t, \rho(\tau)) \nabla \tau\right) \leq f(t)-\sum_{k=0}^{n-1} f^{\nabla^{k}}(b) \widehat{h}_{k}(t, b)
$$




$$
\leq\left\|f^{\nabla^{n}}\right\|_{\infty,[a, b] \cap \mathbb{T}}\left(\int_{t}^{b} \widehat{h}_{n-1}(t, \rho(\tau)) \nabla \tau\right)
$$

$\forall t \in[a, b] \cap \mathbb{T}$.

Consequently we have

$$
\begin{array}{r}
\sum_{k=0}^{n-1} f^{\nabla^{k}}(a) \widehat{h}_{k}(t, a)-\left\|f^{\nabla^{n}}\right\|_{\infty,[a, b] \cap \mathbb{T}} \int_{a}^{t} \widehat{h}_{n-1}(t, \rho(\tau)) \nabla \tau \leq f(t) \\
\leq \sum_{k=0}^{n-1} f^{\nabla^{k}}(a) \widehat{h}_{k}(t, a)+\left\|f^{\nabla^{n}}\right\|_{\infty,[a, b] \cap \mathbb{T}} \int_{a}^{t} \widehat{h}_{n-1}(t, \rho(\tau)) \nabla \tau
\end{array}
$$

and

$$
\begin{aligned}
& \sum_{k=0}^{n-1} f^{\nabla^{k}}(b) \widehat{h}_{k}(t, b)-\left\|f^{\nabla^{n}}\right\|_{\infty,[a, b] \cap \mathbb{T}}\left(\int_{t}^{b} \widehat{h}_{n-1}(t, \rho(\tau)) \nabla \tau\right) \leq f(t) \\
& \quad \leq \sum_{k=0}^{n-1} f^{\nabla^{k}}(b) \widehat{h}_{k}(t, b)+\left\|f^{\nabla^{n}}\right\|_{\infty,[a, b] \cap \mathbb{T}}\left(\int_{t}^{b} \widehat{h}_{n-1}(t, \rho(\tau)) \nabla \tau\right),
\end{aligned}
$$

$\forall t \in[a, b] \cap \mathbb{T}$.

Let any $x \in[a, b] \cap \mathbb{T}$, then integrating (3.11), (3.12) we obtain:

$$
\begin{gathered}
\sum_{k=0}^{n-1} f^{\nabla^{k}}(a) \widehat{h}_{k+1}(x, a)-\left\|f^{\nabla^{n}}\right\|_{\infty,[a, b] \cap \mathbb{T}}\left(\int_{a}^{x}\left(\int_{a}^{t} \widehat{h}_{n-1}(t, \rho(\tau)) \nabla \tau\right) \nabla t\right) \\
\leq \int_{a}^{x} f(t) \nabla t \leq \\
\sum_{k=0}^{n-1} f^{\nabla^{k}}(a) \widehat{h}_{k+1}(x, a)+\left\|f^{\nabla^{n}}\right\|_{\infty,[a, b] \cap \mathbb{T}}\left(\int_{a}^{x}\left(\int_{a}^{t} \widehat{h}_{n-1}(t, \rho(\tau)) \nabla \tau\right) \nabla t\right),
\end{gathered}
$$

and

$$
\begin{gathered}
-\sum_{k=0}^{n-1} f^{\nabla^{k}}(b) \widehat{h}_{k+1}(x, b)-\left\|f^{\nabla^{n}}\right\|_{\infty,[a, b] \cap \mathbb{T}}\left(\int_{x}^{b}\left(\int_{t}^{b} \widehat{h}_{n-1}(t, \rho(\tau)) \nabla \tau\right) \nabla t\right) \\
\leq \int_{x}^{b} f(t) \nabla t \leq \\
-\sum_{k=0}^{n-1} f^{\nabla^{k}}(b) \widehat{h}_{k+1}(x, b)+\left\|f^{\nabla^{n}}\right\|_{\infty,[a, b] \cap \mathbb{T}}\left(\int_{x}^{b}\left(\int_{t}^{b} \widehat{h}_{n-1}(t, \rho(\tau)) \nabla \tau\right) \nabla t\right) .
\end{gathered}
$$


Adding (3.13) and (3.14) we derive

$$
\begin{gathered}
\sum_{k=0}^{n-1}\left(f^{\nabla^{k}}(a) \widehat{h}_{k+1}(x, a)-f^{\nabla^{k}}(b) \widehat{h}_{k+1}(x, b)\right)-\left\|f^{\nabla^{n}}\right\|_{\infty,[a, b] \cap \mathbb{T}} \times \\
{\left[\left(\int_{a}^{x}\left(\int_{a}^{t} \widehat{h}_{n-1}(t, \rho(\tau)) \nabla \tau\right) \nabla t\right)+\left(\int_{x}^{b}\left(\int_{t}^{b} \widehat{h}_{n-1}(t, \rho(\tau)) \nabla \tau\right) \nabla t\right)\right]} \\
\leq \int_{a}^{b} f(t) \nabla t \leq \\
{\left[\left(\sum_{k=0}^{n-1}\left(f^{\nabla^{k}}(a) \widehat{h}_{k+1}(x, a)-f^{\nabla^{k}}(b) \widehat{h}_{k+1}^{t}(x, b)\right)+\left\|f^{\nabla^{n}}\right\|_{\infty,[a, b] \cap \mathbb{T}} \times\right.\right.} \\
\left.\left.\left[\int_{a}(t, \rho(\tau)) \nabla \tau\right) \nabla t\right)+\left(\int_{x}^{b}\left(\int_{t}^{b} \widehat{h}_{n-1}(t, \rho(\tau)) \nabla \tau\right) \nabla t\right)\right]
\end{gathered}
$$

$\forall x \in[a, b] \cap \mathbb{T}$.

The proof is now complete.

We continue with the following result.

Theorem 3.2. Let $f \in C_{l d}^{n}(\mathbb{T}), n \in \mathbb{N}$ is odd, $a, b \in \mathbb{T} ; a \leq b$, where $\mathbb{T}_{k}=\mathbb{T}$. Then 1)

$$
\begin{aligned}
& \left|\int_{a}^{b} f(t) \nabla t-\sum_{k=0}^{n-1}\left(f^{\nabla^{k}}(a) \widehat{h}_{k+1}(x, a)-f^{\nabla^{k}}(b) \widehat{h}_{k+1}(x, b)\right)\right| \leq \\
& \left\|f^{\nabla^{n}}\right\|_{L_{1}([a, b] \cap \mathbb{T})}\left\{\int_{a}^{x}(t-\rho(a))^{n-1} \nabla t+\int_{x}^{b}(\rho(b)-t)^{n-1} \nabla t\right\},
\end{aligned}
$$

$\forall x \in[a, b] \cap \mathbb{T}$,

2) assuming $f^{\nabla^{k}}(a)=f^{\nabla^{k}}(b)=0, k=0,1, \ldots, n-1$, from (3.16) we obtain

$$
\begin{aligned}
\left|\int_{a}^{b} f(t) \nabla t\right| \leq \| & f^{\nabla^{n}} \|_{L_{1}([a, b] \cap \mathbb{T})} \times \\
& \left\{\int_{a}^{x}(t-\rho(a))^{n-1} \nabla t+\int_{x}^{b}(\rho(b)-t)^{n-1} \nabla t\right\},
\end{aligned}
$$

$\forall x \in[a, b] \cap \mathbb{T}$,

$\left.2{ }_{1}\right)$ when $x=a$ by (3.16) we get

$$
\left|\int_{a}^{b} f(t) \nabla t\right| \leq\left\|f^{\nabla^{n}}\right\|_{L_{1}([a, b] \cap \mathbb{T})}\left(\int_{a}^{b}(\rho(b)-t)^{n-1} \nabla t\right),
$$


$\left.2_{2}\right)$ when $x=b$ by (3.16) we get

$$
\left|\int_{a}^{b} f(t) \nabla t\right| \leq\left\|f^{\nabla^{n}}\right\|_{L_{1}([a, b] \cap \mathbb{T})}\left(\int_{a}^{x}(t-\rho(a))^{n-1} \nabla t\right)
$$

$\left.2_{3}\right)$ by (3.18), (3.19) we have

$$
\begin{aligned}
\left|\int_{a}^{b} f(t) \nabla t\right| & \leq\left\|f^{\nabla^{n}}\right\|_{L_{1}([a, b] \cap \mathbb{T})} \times \\
\min & \left\{\left(\int_{a}^{b}(\rho(b)-t)^{n-1} \nabla t\right),\left(\int_{a}^{b}(t-\rho(a))^{n-1} \nabla t\right)\right\},
\end{aligned}
$$

3) assuming $f^{\nabla^{k}}(a)=f^{\nabla^{k}}(b)=0, k=1, \ldots, n-1$, by (3.16) we derive

$$
\begin{gathered}
\left|\int_{a}^{b} f(t) \nabla t-[f(a)(x-a)+f(b)(b-x)]\right| \leq \\
\left\|f^{\nabla^{n}}\right\|_{L_{1}([a, b] \cap \mathbb{T})}\left\{\int_{a}^{x}(t-\rho(a))^{n-1} \nabla t+\int_{x}^{b}(\rho(b)-t)^{n-1} \nabla t\right\},
\end{gathered}
$$

$\forall x \in[a, b] \cap \mathbb{T}$.

Proof. Clearly, here it holds $\left\|f^{\nabla^{n}}\right\|_{L_{1}([a, b] \cap \mathbb{T})}<\infty$.

By Theorem 2.12 we have

$$
f(t)-\sum_{k=0}^{n-1} f^{\nabla^{k}}(a) \widehat{h}_{k}(t, a)=\int_{a}^{t} \widehat{h}_{n-1}(t, \rho(\tau)) f^{\nabla^{n}}(\tau) \nabla \tau
$$

and

$$
f(t)-\sum_{k=0}^{n-1} f^{\nabla^{k}}(b) \widehat{h}_{k}(t, b)=\int_{b}^{t} \widehat{h}_{n-1}(t, \rho(\tau)) f^{\nabla^{n}}(\tau) \nabla \tau,
$$

$\forall t \in[a, b] \cap \mathbb{T}$.

Then

$$
\begin{gathered}
\left|f(t)-\sum_{k=0}^{n-1} f^{\nabla^{k}}(a) \widehat{h}_{k}(t, a)\right|=\left|\int_{a}^{t} \widehat{h}_{n-1}(t, \rho(\tau)) f^{\nabla^{n}}(\tau) \nabla \tau\right| \leq \\
\int_{a}^{t}\left|\widehat{h}_{n-1}(t, \rho(\tau))\right|\left|f^{\nabla^{n}}(\tau)\right| \nabla \tau \leq \int_{a}^{t}|t-\rho(\tau)|^{n-1}\left|f^{\nabla^{n}}(\tau)\right| \nabla \tau \leq \\
(t-\rho(a))^{n-1}\left\|f^{\nabla^{n}}\right\|_{L_{1}([a, b] \cap \mathbb{T})} .
\end{gathered}
$$


Furthermore we have

$$
\begin{gathered}
\left|f(t)-\sum_{k=0}^{n-1} f^{\nabla^{k}}(b) \widehat{h}_{k}(t, b)\right|=\left|\int_{t}^{b} \widehat{h}_{n-1}(t, \rho(\tau)) f^{\nabla^{n}}(\tau) \nabla \tau\right| \leq \\
\int_{t}^{b}\left|\widehat{h}_{n-1}(t, \rho(\tau))\right|\left|f^{\nabla^{n}}(\tau)\right| \nabla \tau \leq \int_{t}^{b}|t-\rho(\tau)|^{n-1}\left|f^{\nabla^{n}}(\tau)\right| \nabla \tau \leq \\
(\rho(b)-t)^{n-1}\left\|f^{\nabla^{n}}\right\|_{L_{1}([a, b] \cap \mathbb{T})} .
\end{gathered}
$$

Therefore it holds

$$
\begin{gathered}
-(t-\rho(a))^{n-1}\left\|f^{\nabla^{n}}\right\|_{L_{1}([a, b] \cap \mathbb{T})} \leq f(t)-\sum_{k=0}^{n-1} f^{\nabla^{k}}(a) \widehat{h}_{k}(t, a) \\
\leq(t-\rho(a))^{n-1}\left\|f^{\nabla^{n}}\right\|_{L_{1}([a, b] \cap \mathbb{T})},
\end{gathered}
$$

$\forall t \in[a, b] \cap \mathbb{T}$, and

$$
\begin{gathered}
-(\rho(b)-t)^{n-1}\left\|f^{\nabla^{n}}\right\|_{L_{1}([a, b] \cap \mathbb{T})} \leq f(t)-\sum_{k=0}^{n-1} f^{\nabla^{k}}(b) \widehat{h}_{k}(t, b) \\
\leq(\rho(b)-t)^{n-1}\left\|f^{\nabla^{n}}\right\|_{L_{1}([a, b] \cap \mathbb{T})}
\end{gathered}
$$

$\forall t \in[a, b] \cap \mathbb{T}$.

Consequently it holds

$$
\begin{gathered}
\sum_{k=0}^{n-1} f^{\nabla^{k}}(a) \widehat{h}_{k}(t, a)-(t-\rho(a))^{n-1}\left\|f^{\nabla^{n}}\right\|_{L_{1}([a, b] \cap \mathbb{T})} \leq f(t) \\
\leq \sum_{k=0}^{n-1} f^{\nabla^{k}}(a) \widehat{h}_{k}(t, a)+(t-\rho(a))^{n-1}\left\|f^{\nabla^{n}}\right\|_{L_{1}([a, b] \cap \mathbb{T})},
\end{gathered}
$$

$\forall t \in[a, b] \cap \mathbb{T}$, and

$$
\begin{gathered}
\sum_{k=0}^{n-1} f^{\nabla^{k}}(b) \widehat{h}_{k}(t, b)-(\rho(b)-t)^{n-1}\left\|f^{\nabla^{n}}\right\|_{L_{1}([a, b] \cap \mathbb{T})} \leq f(t) \\
\leq \sum_{k=0}^{n-1} f^{\nabla^{k}}(b) \widehat{h}_{k}(t, b)+(\rho(b)-t)^{n-1}\left\|f^{\nabla^{n}}\right\|_{L_{1}([a, b] \cap \mathbb{T})},
\end{gathered}
$$

$\forall t \in[a, b] \cap \mathbb{T}$. 
Let any $x \in[a, b] \cap \mathbb{T}$, then by integration we have

$$
\begin{gathered}
\sum_{k=0}^{n-1} f^{\nabla^{k}}(a) \widehat{h}_{k+1}(x, a)-\left(\int_{a}^{x}(t-\rho(a))^{n-1} \nabla t\right)\left\|f^{\nabla^{n}}\right\|_{L_{1}([a, b] \cap \mathbb{T})} \\
\leq \int_{a}^{x} f(t) \nabla t \leq \\
\sum_{k=0}^{n-1} f^{\nabla^{k}}(a) \widehat{h}_{k+1}(x, a)+\left(\int_{a}^{x}(t-\rho(a))^{n-1} \nabla t\right)\left\|f^{\nabla^{n}}\right\|_{L_{1}([a, b] \cap \mathbb{T})},
\end{gathered}
$$

and

$$
\begin{gathered}
-\sum_{k=0}^{n-1} f^{\nabla^{k}}(b) \widehat{h}_{k+1}(x, b)-\left(\int_{x}^{b}(\rho(b)-t)^{n-1} \nabla t\right)\left\|f^{\nabla^{n}}\right\|_{L_{1}([a, b] \cap \mathbb{T})} \\
\leq \int_{x}^{b} f(t) \nabla t \leq \\
-\sum_{k=0}^{n-1} f^{\nabla^{k}}(b) \widehat{h}_{k+1}(x, b)+\left(\int_{x}^{b}(\rho(b)-t)^{n-1} \nabla t\right)\left\|f^{\nabla^{n}}\right\|_{L_{1}([a, b] \cap \mathbb{T})},
\end{gathered}
$$

$\forall x \in[a, b] \cap \mathbb{T}$.

Adding (3.22) and (3.23) we obtain

$$
\begin{aligned}
& \sum_{k=0}^{n-1}\left(f^{\nabla^{k}}(a) \widehat{h}_{k+1}(x, a)-f^{\nabla^{k}}(b) \widehat{h}_{k+1}(x, b)\right)- \\
& \left\|f^{\nabla^{n}}\right\|_{L_{1}([a, b] \cap \mathbb{T})}\left\{\left(\int_{a}^{x}(t-\rho(a))^{n-1} \nabla t\right)+\left(\int_{x}^{b}(\rho(b)-t)^{n-1} \nabla t\right)\right\} \\
& \leq \int_{a}^{b} f(t) \nabla t \leq \\
& \sum_{k=0}^{n-1}\left(f^{\nabla^{k}}(a) \widehat{h}_{k+1}(x, a)-f^{\nabla^{k}}(b) \widehat{h}_{k+1}(x, b)\right)+ \\
& \left\|f^{\nabla^{n}}\right\|_{L_{1}([a, b] \cap \mathbb{T})}\left\{\left(\int_{a}^{x}(t-\rho(a))^{n-1} \nabla t\right)+\left(\int_{x}^{b}(\rho(b)-t)^{n-1} \nabla t\right)\right\},
\end{aligned}
$$

$\forall x \in[a, b] \cap \mathbb{T}$.

The proof is now complete.

We continue with the next result.

Theorem 3.3. Let $f \in C_{l d}^{n}(\mathbb{T}), n \in \mathbb{N}$ is odd, $a, b \in \mathbb{T} ; a \leq b ; p, q>1: \frac{1}{p}+\frac{1}{q}=1$. We suppose Assumptions 2.19, 2.21. Then 
1)

$$
\begin{aligned}
& \left|\int_{a}^{b} f(t) \nabla t-\sum_{k=0}^{n-1}\left(f^{\nabla^{k}}(a) \widehat{h}_{k+1}(x, a)-f^{\nabla^{k}}(b) \widehat{h}_{k+1}(x, b)\right)\right| \\
& \leq\left\|f^{\nabla^{n}}\right\|_{L_{q}([a, b] \cap \mathbb{T})} \times \\
& {\left[\int_{a}^{x}\left(\int_{a}^{t} \widehat{h}_{n-1}(t, \rho(\tau))^{p} \nabla \tau\right)^{\frac{1}{p}} \nabla t+\int_{x}^{b}\left(\int_{t}^{b} \widehat{h}_{n-1}(t, \rho(\tau))^{p} \nabla \tau\right)^{\frac{1}{p}} \nabla t\right],}
\end{aligned}
$$

$\forall x \in[a, b] \cap \mathbb{T}$,

2) assuming $f^{\nabla^{k}}(a)=f^{\nabla^{k}}(b)=0, k=0,1, \ldots, n-1$, by (3.25) we have that

$$
\begin{aligned}
& \left|\int_{a}^{b} f(t) \nabla t\right| \leq\left\|f^{\nabla^{n}}\right\|_{L_{q}([a, b] \cap \mathbb{T})} \times \\
& {\left[\int_{a}^{x}\left(\int_{a}^{t} \widehat{h}_{n-1}(t, \rho(\tau))^{p} \nabla \tau\right)^{\frac{1}{p}} \nabla t+\int_{x}^{b}\left(\int_{t}^{b} \widehat{h}_{n-1}(t, \rho(\tau))^{p} \nabla \tau\right)^{\frac{1}{p}} \nabla t\right],}
\end{aligned}
$$

$\forall x \in[a, b] \cap \mathbb{T}$,

21) when $x=a$ by (3.26) we get

$$
\left|\int_{a}^{b} f(t) \nabla t\right| \leq\left\|f^{\nabla^{n}}\right\|_{L_{q}([a, b] \cap \mathbb{T})}\left(\int_{a}^{b}\left(\int_{t}^{b} \widehat{h}_{n-1}(t, \rho(\tau))^{p} \nabla \tau\right)^{\frac{1}{p}} \nabla t\right),
$$

$\left.2_{2}\right)$ when $x=b$ by (3.26) we get

$$
\left|\int_{a}^{b} f(t) \nabla t\right| \leq\left\|f^{\nabla^{n}}\right\|_{L_{q}([a, b] \cap \mathbb{T})}\left(\int_{a}^{b}\left(\int_{a}^{t} \widehat{h}_{n-1}(t, \rho(\tau))^{p} \nabla \tau\right)^{\frac{1}{p}} \nabla t\right),
$$

$\left.2_{3}\right)$ by (3.27), (3.28) we derive that

$$
\begin{gathered}
\left|\int_{a}^{b} f(t) \nabla t\right| \leq\left\|f^{\nabla^{n}}\right\|_{L_{q}([a, b] \cap \mathbb{T})} \times \\
\min \left\{\int_{a}^{b}\left(\int_{t}^{b} \widehat{h}_{n-1}(t, \rho(\tau))^{p} \nabla \tau\right)^{\frac{1}{p}} \nabla t, \int_{a}^{b}\left(\int_{a}^{t} \widehat{h}_{n-1}(t, \rho(\tau))^{p} \nabla \tau\right)^{\frac{1}{p}} \nabla t\right\},
\end{gathered}
$$


3) assuming $f^{\nabla^{k}}(a)=f^{\nabla^{k}}(b)=0, k=1, \ldots, n-1$, by (3.25) we obtain

$$
\begin{aligned}
& \left|\int_{a}^{b} f(t) \nabla t-[f(a)(x-a)+f(b)(b-x)]\right| \leq\left\|f^{\nabla^{n}}\right\|_{L_{q}([a, b] \cap \mathbb{T})} \times \\
& {\left[\int_{a}^{x}\left(\int_{a}^{t} \widehat{h}_{n-1}(t, \rho(\tau))^{p} \nabla \tau\right)^{\frac{1}{p}} \nabla t+\int_{x}^{b}\left(\int_{t}^{b} \widehat{h}_{n-1}(t, \rho(\tau))^{p} \nabla \tau\right)^{\frac{1}{p}} \nabla t\right],}
\end{aligned}
$$

$\forall x \in[a, b] \cap \mathbb{T}$.

Proof. As before we have

$$
K(t, a):=f(t)-\sum_{k=0}^{n-1} f^{\nabla^{k}}(a) \widehat{h}_{k}(t, a)=\int_{a}^{t} \widehat{h}_{n-1}(t, \rho(\tau)) f^{\nabla^{n}}(\tau) \nabla \tau,
$$

and

$$
K(t, b):=f(t)-\sum_{k=0}^{n-1} f^{\nabla^{k}}(b) \widehat{h}_{k}(t, b)=\int_{b}^{t} \widehat{h}_{n-1}(t, \rho(\tau)) f^{\nabla^{n}}(\tau) \nabla \tau,
$$

$\forall t \in[a, b] \cap \mathbb{T}$.

We have that (by use of Theorem 2.15)

$$
\begin{aligned}
|K(t, a)| & \leq\left(\int_{a}^{t} \widehat{h}_{n-1}(t, \rho(\tau))^{p} \nabla \tau\right)^{\frac{1}{p}}\left(\int_{a}^{t}\left|f^{\nabla^{n}}(\tau)\right|^{q} \nabla \tau\right)^{\frac{1}{q}} \\
& \leq\left(\int_{a}^{t} \widehat{h}_{n-1}(t, \rho(\tau))^{p} \nabla \tau\right)^{\frac{1}{p}}\left\|f^{\nabla^{n}}\right\|_{L_{q}([a, b] \cap \mathbb{T})},
\end{aligned}
$$

and

$$
\begin{gathered}
|K(t, b)|=\left|\int_{t}^{b} \widehat{h}_{n-1}(t, \rho(\tau)) f^{\nabla^{n}}(\tau) \nabla \tau\right| \leq \\
\left(\int_{t}^{b} \widehat{h}_{n-1}(t, \rho(\tau))^{p} \nabla \tau\right)^{\frac{1}{p}}\left(\int_{t}^{b}\left|f^{\nabla^{n}}(\tau)\right|^{q} \nabla \tau\right)^{\frac{1}{q}} \\
\leq\left(\int_{t}^{b} \widehat{h}_{n-1}(t, \rho(\tau))^{p} \nabla \tau\right)^{\frac{1}{p}}\left\|f^{\nabla^{n}}\right\|_{L_{q}([a, b] \cap \mathbb{T})},
\end{gathered}
$$

$\forall t \in[a, b] \cap \mathbb{T}$.

Hence it holds

$$
-\left(\int_{a}^{t} \widehat{h}_{n-1}(t, \rho(\tau))^{p} \nabla \tau\right)^{\frac{1}{p}}\left\|f^{\nabla^{n}}\right\|_{L_{q}([a, b] \cap \mathbb{T})} \leq K(t, a)
$$




$$
\leq\left(\int_{a}^{t} \widehat{h}_{n-1}(t, \rho(\tau))^{p} \nabla \tau\right)^{\frac{1}{p}}\left\|f^{\nabla^{n}}\right\|_{L_{q}([a, b] \cap \mathbb{T})}
$$

and

$$
\begin{gathered}
-\left(\int_{t}^{b} \widehat{h}_{n-1}(t, \rho(\tau))^{p} \nabla \tau\right)^{\frac{1}{p}}\left\|f^{\nabla^{n}}\right\|_{L_{q}([a, b] \cap \mathbb{T})} \leq K(t, b) \\
\leq\left(\int_{t}^{b} \widehat{h}_{n-1}(t, \rho(\tau))^{p} \nabla \tau\right)^{\frac{1}{p}}\left\|f^{\nabla^{n}}\right\|_{L_{q}([a, b] \cap \mathbb{T})},
\end{gathered}
$$

$\forall t \in[a, b] \cap \mathbb{T}$.

That is

$$
\begin{gathered}
\sum_{k=0}^{n-1} f^{\nabla^{k}}(a) \widehat{h}_{k}(t, a)-\left(\int_{a}^{t} \widehat{h}_{n-1}(t, \rho(\tau))^{p} \nabla \tau\right)^{\frac{1}{p}}\left\|f^{\nabla^{n}}\right\|_{L_{q}([a, b] \cap \mathbb{T})} \leq f(t) \\
\leq \sum_{k=0}^{n-1} f^{\nabla^{k}}(a) \widehat{h}_{k}(t, a)+\left(\int_{a}^{t} \widehat{h}_{n-1}(t, \rho(\tau))^{p} \nabla \tau\right)^{\frac{1}{p}}\left\|f^{\nabla^{n}}\right\|_{L_{q}([a, b] \cap \mathbb{T})}
\end{gathered}
$$

and

$$
\begin{gathered}
\sum_{k=0}^{n-1} f^{\nabla^{k}}(b) \widehat{h}_{k}(t, b)-\left(\int_{t}^{b} \widehat{h}_{n-1}(t, \rho(\tau))^{p} \nabla \tau\right)^{\frac{1}{p}}\left\|f^{\nabla^{n}}\right\|_{L_{q}([a, b] \cap \mathbb{T})} \leq f(t) \\
\leq \sum_{k=0}^{n-1} f^{\nabla^{k}}(b) \widehat{h}_{k}(t, b)+\left(\int_{t}^{b} \widehat{h}_{n-1}(t, \rho(\tau))^{p} \nabla \tau\right)^{\frac{1}{p}}\left\|f^{\nabla^{n}}\right\|_{L_{q}([a, b] \cap \mathbb{T})}
\end{gathered}
$$

$\forall t \in[a, b] \cap \mathbb{T}$.

Let any $x \in[a, b] \cap \mathbb{T}$, then by integration we get

$$
\begin{gathered}
\sum_{k=0}^{n-1} f^{\nabla^{k}}(a) \widehat{h}_{k+1}(x, a)-\left\|f^{\nabla^{n}}\right\|_{L_{q}([a, b] \cap \mathbb{T})}\left(\int_{a}^{x}\left(\int_{a}^{t} \widehat{h}_{n-1}(t, \rho(\tau))^{p} \nabla \tau\right)^{\frac{1}{p}} \nabla t\right) \\
\leq \int_{a}^{x} f(t) \nabla t \leq \\
\sum_{k=0}^{n-1} f^{\nabla^{k}}(a) \widehat{h}_{k+1}(x, a)+\left\|f^{\nabla^{n}}\right\|_{L_{q}([a, b] \cap \mathbb{T})}\left(\int_{a}^{x}\left(\int_{a}^{t} \widehat{h}_{n-1}(t, \rho(\tau))^{p} \nabla \tau\right)^{\frac{1}{p}} \nabla t\right),
\end{gathered}
$$

and

$$
-\sum_{k=0}^{n-1} f^{\nabla^{k}}(b) \widehat{h}_{k+1}(x, b)-\left\|f^{\nabla^{n}}\right\|_{L_{q}([a, b] \cap \mathbb{T})}\left(\int_{x}^{b}\left(\int_{t}^{b} \widehat{h}_{n-1}(t, \rho(\tau))^{p} \nabla \tau\right)^{\frac{1}{p}} \nabla t\right)
$$




$$
\begin{gathered}
\leq \int_{x}^{b} f(t) \nabla t \leq \\
-\sum_{k=0}^{n-1} f^{\nabla^{k}}(b) \widehat{h}_{k+1}(x, b)+\left\|f^{\nabla^{n}}\right\|_{L_{q}([a, b] \cap \mathbb{T})}\left(\int_{x}^{b}\left(\int_{t}^{b} \widehat{h}_{n-1}(t, \rho(\tau))^{p} \nabla \tau\right)^{\frac{1}{p}} \nabla t\right) .
\end{gathered}
$$

Adding (3.31) and (3.32) we obtain

$$
\begin{gathered}
\sum_{k=0}^{n-1}\left(f^{\nabla^{k}}(a) \widehat{h}_{k+1}(x, a)-f^{\nabla^{k}}(b) \widehat{h}_{k+1}(x, b)\right)- \\
\left\|f^{\nabla^{n}}\right\|_{L_{q}([a, b] \cap \mathbb{T})}\left\{\left(\int_{a}^{x}\left(\int_{a}^{t} \widehat{h}_{n-1}(t, \rho(\tau))^{p} \nabla \tau\right)^{\frac{1}{p}} \nabla t\right)+\right. \\
\left.\left(\int_{x}^{b}\left(\int_{t}^{b} \widehat{h}_{n-1}(t, \rho(\tau))^{p} \nabla \tau\right)^{\frac{1}{p}} \nabla t\right)\right\} \\
\leq \int_{a}^{b} f(t) \nabla t \leq \\
\sum_{k=0}^{n-1}\left(f^{\nabla^{k}}(a) \widehat{h}_{k+1}(x, a)-f^{\nabla^{k}}(b) \widehat{h}_{k+1}(x, b)\right)+ \\
\left\|f^{\nabla^{n}}\right\|_{L_{q}([a, b] \cap \mathbb{T})}\left\{\left(\int_{a}^{x}\left(\int_{a}^{t} \widehat{h}_{n-1}(t, \rho(\tau))^{p} \nabla \tau\right)^{\frac{1}{p}} \nabla t\right)+\right. \\
\left.\left(\int_{x}^{b}\left(\int_{t}^{b} \widehat{h}_{n-1}(t, \rho(\tau))^{p} \nabla \tau\right)^{\frac{1}{p}} \nabla t\right)\right\}
\end{gathered}
$$

$\forall x \in[a, b] \cap \mathbb{T}$.

The proof is now complete.

We give the next result.

Theorem 3.4. Let $f \in C_{l d}^{n}(\mathbb{T}), m, n \in \mathbb{N}, m<n, n-m$ is odd, $a, b \in \mathbb{T} ; a \leq b$. Here $\rho$ is continuous and $\widehat{h}_{n-m-1}(t, s)$ is jointly continuous. Also assume $\mathbb{T}_{k}=\mathbb{T}$. Then

1)

$$
\begin{gathered}
\left|\int_{a}^{b} f^{\nabla^{m}}(t) \nabla t-\left(\sum_{k=0}^{n-m-1}\left(f^{\nabla^{k+m}}(a) \widehat{h}_{k+1}(x, a)-f^{\nabla^{k+m}}(b) \widehat{h}_{k+1}(x, b)\right)\right)\right| \leq \\
\left\|f^{\nabla^{n}}\right\|_{\infty,[a, b] \cap \mathbb{T}}\left[\left(\int_{a}^{x}\left(\int_{a}^{t} \widehat{h}_{n-m-1}(t, \rho(\tau)) \nabla \tau\right) \nabla t\right)+\right.
\end{gathered}
$$




$$
\left.\left(\int_{x}^{b}\left(\int_{t}^{b} \widehat{h}_{n-m-1}(t, \rho(\tau)) \nabla \tau\right) \nabla t\right)\right]
$$

$\forall x \in[a, b] \cap \mathbb{T}$,

2) assuming $f^{\nabla^{k+m}}(a)=f^{\nabla^{k+m}}(b)=0, k=0,1, \ldots, n-m-1$, we get from (3.34) that

$$
\begin{aligned}
& \left|\int_{a}^{b} f^{\nabla^{m}}(t) \nabla t\right| \leq\left\|f^{\nabla^{n}}\right\|_{\infty,[a, b] \cap \mathbb{T}} \times \\
& {\left[\int_{a}^{x}\left(\int_{a}^{t} \widehat{h}_{n-m-1}(t, \rho(\tau)) \nabla \tau\right) \nabla t+\int_{x}^{b}\left(\int_{t}^{b} \widehat{h}_{n-m-1}(t, \rho(\tau)) \nabla \tau\right) \nabla t\right],}
\end{aligned}
$$

$\forall x \in[a, b] \cap \mathbb{T}$,

21) when $x=a$ we get from (3.35) that

$$
\left|\int_{a}^{b} f^{\nabla^{m}}(t) \nabla t\right| \leq\left\|f^{\nabla^{n}}\right\|_{\infty,[a, b] \cap \mathbb{T}}\left(\int_{a}^{b}\left(\int_{t}^{b} \widehat{h}_{n-m-1}(t, \rho(\tau)) \nabla \tau\right) \nabla t\right),
$$

$2_{2}$ ) when $x=b$ we get from (3.35) that

$$
\left|\int_{a}^{b} f^{\nabla^{m}}(t) \nabla t\right| \leq\left\|f^{\nabla^{n}}\right\|_{\infty,[a, b] \cap \mathbb{T}}\left(\int_{a}^{b}\left(\int_{a}^{t} \widehat{h}_{n-m-1}(t, \rho(\tau)) \nabla \tau\right) \nabla t\right),
$$

$\left.2_{3}\right)$ by (3.36), (3.37) we get

$$
\begin{aligned}
& \left|\int_{a}^{b} f^{\nabla^{m}}(t) \nabla t\right| \leq\left\|f^{\nabla^{n}}\right\|_{\infty,[a, b] \cap \mathbb{T}} \times \\
& \min \left\{\int_{a}^{b}\left(\int_{t}^{b} \widehat{h}_{n-m-1}(t, \rho(\tau)) \nabla \tau\right) \nabla t, \int_{a}^{b}\left(\int_{a}^{t} \widehat{h}_{n-m-1}(t, \rho(\tau)) \nabla \tau\right) \nabla t\right\},
\end{aligned}
$$

and

3) assuming $f^{\nabla^{k+m}}(a)=f^{\nabla^{k+m}}(b)=0, k=1, \ldots, n-m-1$, from (3.34) we obtain

$$
\begin{gathered}
\left|\int_{a}^{b} f^{\nabla^{m}}(t) \nabla t-\left[f^{\nabla^{m}}(a)(x-a)+f^{\nabla^{m}}(b)(b-x)\right]\right| \leq\left\|f^{\nabla^{n}}\right\|_{\infty,[a, b] \cap \mathbb{T}} \times \\
{\left[\int_{a}^{x}\left(\int_{a}^{t} \widehat{h}_{n-m-1}(t, \rho(\tau)) \nabla \tau\right) \nabla t+\int_{x}^{b}\left(\int_{t}^{b} \widehat{h}_{n-m-1}(t, \rho(\tau)) \nabla \tau\right) \nabla t\right],}
\end{gathered}
$$

$\forall x \in[a, b] \cap \mathbb{T}$. 
Proof. As in the proof of Theorem 3.1, now using Corollary 2.13.

We give the following theorem.

Theorem 3.5. Let $f \in C_{l d}^{n}(\mathbb{T}), m, n \in \mathbb{N}, m<n, n-m$ is odd, $a, b \in \mathbb{T}$; $a \leq b$, where $\mathbb{T}_{k}=\mathbb{T}$. Then

1)

$$
\begin{gathered}
\left|\int_{a}^{b} f^{\nabla^{m}}(t) \nabla t-\sum_{k=0}^{n-m-1}\left(f^{\nabla^{k+m}}(a) \widehat{h}_{k+1}(x, a)-f^{\nabla^{k+m}}(b) \widehat{h}_{k+1}(x, b)\right)\right| \leq \\
\left\|f^{\nabla^{n}}\right\|_{L_{1}([a, b] \cap \mathbb{T})}\left\{\int_{a}^{x}(t-\rho(a))^{n-m-1} \nabla t+\int_{x}^{b}(\rho(b)-t)^{n-m-1} \nabla t\right\},
\end{gathered}
$$

$\forall x \in[a, b] \cap \mathbb{T}$,

2) assuming $f^{\nabla^{k+m}}(a)=f^{\nabla^{k+m}}(b)=0, k=0,1, \ldots, n-m-1$, we get from (3.40) that

$$
\begin{aligned}
\left|\int_{a}^{b} f^{\nabla^{m}}(t) \nabla t\right| & \leq\left\|f^{\nabla^{n}}\right\|_{L_{1}([a, b] \cap \mathbb{T})} \times \\
& \left\{\int_{a}^{x}(t-\rho(a))^{n-m-1} \nabla t+\int_{x}^{b}(\rho(b)-t)^{n-m-1} \nabla t\right\},
\end{aligned}
$$

$\forall x \in[a, b] \cap \mathbb{T}$,

21) when $x=a$ by (3.41) we get

$$
\left|\int_{a}^{b} f^{\nabla^{m}}(t) \nabla t\right| \leq\left\|f^{\nabla^{n}}\right\|_{L_{1}([a, b] \cap \mathbb{T})}\left(\int_{a}^{b}(\rho(b)-t)^{n-m-1} \nabla t\right),
$$

2.) when $x=b$ by (3.41) we get

$$
\left|\int_{a}^{b} f^{\nabla^{m}}(t) \nabla t\right| \leq\left\|f^{\nabla^{n}}\right\|_{L_{1}([a, b] \cap \mathbb{T})}\left(\int_{a}^{x}(t-\rho(a))^{n-m-1} \nabla t\right),
$$

$2_{3}$ ) by (3.42), (3.43) we have

$$
\begin{aligned}
& \left|\int_{a}^{b} f^{\nabla^{m}}(t) \nabla t\right| \leq\left\|f^{\nabla^{n}}\right\|_{L_{1}([a, b] \cap \mathbb{T})} \times \\
& \quad \min \left\{\left(\int_{a}^{b}(\rho(b)-t)^{n-m-1} \nabla t\right),\left(\int_{a}^{b}(t-\rho(a))^{n-m-1} \nabla t\right)\right\},
\end{aligned}
$$

and 
3) assuming $f^{\nabla^{k+m}}(a)=f^{\nabla^{k+m}}(b)=0, k=1, \ldots, n-m-1$, from (3.40) we obtain

$$
\begin{gathered}
\left|\int_{a}^{b} f^{\nabla^{m}}(t) \nabla t-\left[f^{\nabla^{m}}(a)(x-a)+f^{\nabla^{m}}(b)(b-x)\right]\right| \leq \\
\left\|f^{\nabla^{n}}\right\|_{L_{1}([a, b] \cap \mathbb{T})}\left\{\int_{a}^{x}(t-\rho(a))^{n-m-1} \nabla t+\int_{x}^{b}(\rho(b)-t)^{n-m-1} \nabla t\right\},
\end{gathered}
$$

$\forall x \in[a, b] \cap \mathbb{T}$.

Proof. As in Theorem 3.2, now using Corollary 2.13.

We also give the next result.

Theorem 3.6. Let $f \in C_{l d}^{n}(\mathbb{T}), m, n \in \mathbb{N}, m<n, n-m$ is odd, $a, b \in \mathbb{T}$; $a \leq b$. Let also $p, q>1: \frac{1}{p}+\frac{1}{q}=1$. We suppose Assumptions 2.19, 2.22. Then

1)

$$
\begin{gathered}
\left|\int_{a}^{b} f^{\nabla^{m}}(t) \nabla t-\sum_{k=0}^{n-m-1}\left(f^{\nabla^{k+m}}(a) \widehat{h}_{k+1}(x, a)-f^{\nabla^{k+m}}(b) \widehat{h}_{k+1}(x, b)\right)\right| \leq \\
\left\|f^{\nabla^{n}}\right\|_{L_{q}([a, b] \cap \mathbb{T})}\left[\left(\int_{a}^{x}\left(\int_{a}^{t} \widehat{h}_{n-m-1}(t, \rho(\tau))^{p} \nabla \tau\right)^{\frac{1}{p}} \nabla t\right)+\right. \\
\left.\left(\int_{x}^{b}\left(\int_{t}^{b} \widehat{h}_{n-m-1}(t, \rho(\tau))^{p} \nabla \tau\right)^{\frac{1}{p}} \nabla t\right)\right]
\end{gathered}
$$

$\forall x \in[a, b] \cap \mathbb{T}$,

2) assuming $f^{\nabla^{k+m}}(a)=f^{\nabla^{k+m}}(b)=0, k=0,1, \ldots, n-m-1$, we get from (3.46) that

$$
\begin{aligned}
\left|\int_{a}^{b} f^{\nabla^{m}}(t) \nabla t\right| \leq & \left\|f^{\nabla^{n}}\right\|_{L_{q}([a, b] \cap \mathbb{T})}\left[\left(\int_{a}^{x}\left(\int_{a}^{t} \widehat{h}_{n-m-1}(t, \rho(\tau))^{p} \nabla \tau\right)^{\frac{1}{p}} \nabla t\right)+\right. \\
& \left.\left(\int_{x}^{b}\left(\int_{t}^{b} \widehat{h}_{n-m-1}(t, \rho(\tau))^{p} \nabla \tau\right)^{\frac{1}{p}} \nabla t\right)\right]
\end{aligned}
$$

$\forall x \in[a, b] \cap \mathbb{T}$, 
$\left.2{ }_{1}\right)$ when $x=a$ we get from (3.47) that

$$
\left|\int_{a}^{b} f^{\nabla^{m}}(t) \nabla t\right| \leq\left\|f^{\nabla^{n}}\right\|_{L_{q}([a, b] \cap \mathbb{T})}\left(\int_{a}^{b}\left(\int_{t}^{b} \widehat{h}_{n-m-1}(t, \rho(\tau))^{p} \nabla \tau\right)^{\frac{1}{p}} \nabla t\right),
$$

$2_{2}$ ) when $x=b$ we get from (3.47) that

$$
\left|\int_{a}^{b} f^{\nabla^{m}}(t) \nabla t\right| \leq\left\|f^{\nabla^{n}}\right\|_{L_{q}([a, b] \cap \mathbb{T})}\left(\int_{a}^{b}\left(\int_{a}^{t} \widehat{h}_{n-m-1}(t, \rho(\tau))^{p} \nabla \tau\right)^{\frac{1}{p}} \nabla t\right),
$$

$\left.2_{3}\right)$ by (3.48), (3.49) we get

$$
\begin{aligned}
\left|\int_{a}^{b} f^{\nabla^{m}}(t) \nabla t\right| \leq & \left\|f^{\nabla^{n}}\right\|_{L_{q}([a, b] \cap \mathbb{T})} \times \\
\min \{ & \left(\int_{a}^{b}\left(\int_{t}^{b} \widehat{h}_{n-m-1}(t, \rho(\tau))^{p} \nabla \tau\right)^{\frac{1}{p}} \nabla t\right), \\
& \left.\left(\int_{a}^{b}\left(\int_{a}^{t} \widehat{h}_{n-m-1}(t, \rho(\tau))^{p} \nabla \tau\right)^{\frac{1}{p}} \nabla t\right)\right\},
\end{aligned}
$$

and

3) assuming $f^{\nabla^{k+m}}(a)=f^{\nabla^{k+m}}(b)=0, k=1, \ldots, n-m-1$, we get from (3.46) that

$$
\begin{gathered}
\left|\int_{a}^{b} f^{\nabla^{m}}(t) \nabla t-\left[f^{\nabla^{m}}(a)(x-a)+f^{\nabla^{m}}(b)(b-x)\right]\right| \leq \\
\left\|f^{\nabla^{n}}\right\|_{L_{q}([a, b] \cap \mathbb{T})}\left[\left(\int_{a}^{x}\left(\int_{a}^{t} \widehat{h}_{n-m-1}(t, \rho(\tau))^{p} \nabla \tau\right)^{\frac{1}{p}} \nabla t\right)+\right. \\
\left.\left(\int_{x}^{b}\left(\int_{t}^{b} \widehat{h}_{n-m-1}(t, \rho(\tau))^{p} \nabla \tau\right)^{\frac{1}{p}} \nabla t\right)\right],
\end{gathered}
$$

$\forall x \in[a, b] \cap \mathbb{T}$.

Proof. As in Theorem 3.3, by using Corollary 2.13.

\section{Applications}

Next we give applications of our initial main results. 
Theorem 4.1. Let $f \in C^{n}([a, b]), n \in \mathbb{N}$ is odd and $[a, b] \subset \mathbb{R}$. Then

$$
\begin{gathered}
\left|\int_{a}^{b} f(t) d t-\sum_{k=0}^{n-1} \frac{1}{(k+1) !}\left(f^{(k)}(a)(x-a)^{k+1}+(-1)^{k} f^{(k)}(b)(b-x)^{k+1}\right)\right| \\
\leq \frac{\left\|f^{(n)}\right\|_{\infty,[a, b]}}{(n+1) !}\left[(x-a)^{n+1}+(b-x)^{n+1}\right]
\end{gathered}
$$

$\forall x \in[a, b]$.

Proof. By Theorem 3.1, (3.1).

We continue with the following.

Theorem 4.2. Let $f \in C^{n}([a, b]), n \in \mathbb{N}$ is odd, $[a, b] \subset \mathbb{R}$. Then

$$
\begin{gathered}
\left|\int_{a}^{b} f(t) d t-\sum_{k=0}^{n-1} \frac{1}{(k+1) !}\left(f^{(k)}(a)(x-a)^{k+1}+(-1)^{k} f^{(k)}(b)(b-x)^{k+1}\right)\right| \\
\leq \frac{\left\|f^{(n)}\right\|_{L_{1}([a, b])}}{n}\left[(x-a)^{n}+(b-x)^{n}\right]
\end{gathered}
$$

$\forall x \in[a, b]$.

Proof. By Theorem 3.2, (3.16).

We also give the next result.

Theorem 4.3. Let $f \in C^{n}([a, b]), n \in \mathbb{N}$ is odd and $[a, b] \subset \mathbb{R}$. Let also $p, q>1$ : $\frac{1}{p}+\frac{1}{q}=1$. Then

$$
\begin{gathered}
\left|\int_{a}^{b} f(t) d t-\sum_{k=0}^{n-1} \frac{1}{(k+1) !}\left(f^{(k)}(a)(x-a)^{k+1}+(-1)^{k} f^{(k)}(b)(b-x)^{k+1}\right)\right| \\
\quad \leq \frac{\left\|f^{(n)}\right\|_{L_{q}([a, b])}}{(n-1) !(p(n-1)+1)^{\frac{1}{p}}\left(n+\frac{1}{p}\right)}\left[(x-a)^{n+\frac{1}{p}}+(b-x)^{n+\frac{1}{p}}\right],
\end{gathered}
$$

$\forall x \in[a, b]$.

Proof. By Theorem 3.3, (3.25).

We continue with the following theorem. 
Theorem 4.4. Let $f: \mathbb{Z} \rightarrow \mathbb{R}$, $n$ is an odd number, $a, b \in \mathbb{Z} ; a \leq b$. Then

$$
\begin{gathered}
\left|\sum_{t=a+1}^{b} f(t)-\sum_{k=0}^{n-1} \frac{1}{(k+1) !}\left(\nabla^{k} f(a)(x-a)^{(\overline{k+1})}-\nabla^{k} f(b)(x-b)^{(\overline{k+1})}\right)\right| \leq \\
\frac{\left\|\nabla^{n} f\right\|_{\infty,[a, b] \cap \mathbb{Z}}}{(n-1) !}\left[\left(\sum_{t=a+1}^{x}\left(\sum_{\tau=a+1}^{t}(t-\tau+1)^{(\overline{n-1})}\right)\right)+\right. \\
\left.\left(\sum_{t=x+1}^{b}\left(\sum_{\tau=t+1}^{b}(t-\tau+1)^{(\overline{n-1})}\right)\right)\right]
\end{gathered}
$$

$\forall x \in[a, b] \cap \mathbb{Z}$.

Proof. By Theorem 3.1, (3.1).

We give the next result.

Theorem 4.5. Let $f: \mathbb{Z} \rightarrow \mathbb{R}, n \in \mathbb{N}$ is odd, $a, b \in \mathbb{Z} ; a \leq b$. Then

$$
\begin{gathered}
\left|\sum_{t=a+1}^{b} f(t)-\sum_{k=0}^{n-1} \frac{1}{(k+1) !}\left(\nabla^{k} f(a)(x-a)^{(\overline{k+1})}-\nabla^{k} f(b)(x-b)^{(\overline{k+1})}\right)\right| \leq \\
\quad\left(\sum_{t=a+1}^{b}\left|\nabla^{n} f(t)\right|\right)\left\{\sum_{t=a+1}^{x}(t-a+1)^{\overline{n-1}}+\sum_{t=x+1}^{b}(b-1-t)^{\overline{n-1}}\right\},
\end{gathered}
$$

$\forall x \in[a, b] \cap \mathbb{Z}$.

Proof. By Theorem 3.2, (3.16).

We give the next theorem.

Theorem 4.6. Let $f: \mathbb{Z} \rightarrow \mathbb{R}, n$ is an odd number, $a, b \in \mathbb{Z} ; a \leq b$, let also $p, q>1$ : $\frac{1}{p}+\frac{1}{q}=1$. Then

$$
\begin{gathered}
\left|\sum_{t=a+1}^{b} f(t)-\sum_{k=0}^{n-1} \frac{1}{(k+1) !}\left(\nabla^{k} f(a)(x-a)^{(\overline{k+1})}-\nabla^{k} f(b)(x-b)^{(\overline{k+1})}\right)\right| \leq \\
\frac{\left(\sum_{t=a+1}^{b}\left|\nabla^{n} f(t)\right|^{q}\right)^{\frac{1}{q}}}{(n-1) !}\left[\left(\sum_{t=a+1}^{x}\left(\sum_{\tau=a+1}^{t}\left((t-\tau+1)^{(\overline{n-1})}\right)^{p}\right)^{\frac{1}{p}}\right)+\right. \\
\left.\left(\sum_{t=x+1}^{b}\left(\sum_{\tau=t+1}^{b}\left((t-\tau+1)^{(\overline{n-1})}\right)^{p}\right)^{\frac{1}{p}}\right)\right],
\end{gathered}
$$

$\forall x \in[a, b] \cap \mathbb{Z}$. 
Proof. By Theorem 3.3, (3.25).

We need the following remark.

Remark 4.7 (See [4]). We consider the time scale $\mathbb{T}=q^{\overline{\mathbb{Z}}}=\left\{0,1, q, q^{-1}, q^{2}, q^{-2}, \ldots\right\}$, for some $q>1$. Here $\rho(t)=\frac{t}{q}, \forall t \in \mathbb{T}$. We have that

$$
\widehat{h}_{k}(t, s)=\prod_{r=0}^{k-1} \frac{q^{r} t-s}{\sum_{j=0}^{r} q^{j}}, \text { for all } s, t \in \mathbb{T},
$$

for all $k \in \mathbb{N}_{0}$.

We continue with the next theorem.

Theorem 4.8. Let $f \in C_{l d}^{n}\left(q^{\bar{Z}}\right), n \in \mathbb{N}$ is odd, $a, b \in q^{\bar{Z}} ; a \leq b$. Then

$$
\begin{gathered}
\left|\int_{a}^{b} f(t) \nabla t-\sum_{k=0}^{n-1}\left(f^{\nabla^{k}}(a) \prod_{\nu=0}^{k} \frac{q^{\nu} x-a}{\sum_{\mu=0}^{\nu} q^{\mu}}-f^{\nabla^{k}}(b) \prod_{\nu=0}^{k} \frac{q^{\nu} x-b}{\sum_{\mu=0}^{\nu} q^{\mu}}\right)\right| \leq \\
\left\|f^{\nabla^{n}}\right\|_{L_{1}\left([a, b] \cap q^{\bar{Z}}\right)}\left\{\int_{a}^{x}\left(t-\frac{a}{q}\right)^{n-1} \nabla t+\int_{x}^{b}\left(\frac{b}{q}-t\right)^{n-1} \nabla t\right\},
\end{gathered}
$$

$\forall x \in[a, b] \cap q^{\overline{\mathbb{Z}}}$.

Proof. By Theorem 3.2, (3.16).

We finish with the next theorem.

Theorem 4.9. Let $f \in C_{l d}^{n}\left(q^{\overline{\mathbb{Z}}}\right), m, n \in \mathbb{N} ; m<n, n-m$ is odd, $a, b \in q^{\overline{\mathbb{Z}}} ; a \leq b$. Then

$$
\begin{gathered}
\left|\int_{a}^{b} f^{\nabla^{m}}(t) \nabla t-\sum_{k=0}^{n-m-1}\left(f^{\nabla^{k+m}}(a) \prod_{\nu=0}^{k} \frac{q^{\nu} x-a}{\sum_{\mu=0}^{\nu} q^{\mu}}-f^{\nabla^{k+m}}(b) \prod_{\nu=0}^{k} \frac{q^{\nu} x-b}{\sum_{\mu=0}^{\nu} q^{\mu}}\right)\right| \leq \\
\left\|f^{\nabla^{n}}\right\|_{L_{1}\left([a, b] \cap q^{\bar{Z}}\right)}\left\{\int_{a}^{x}\left(t-\frac{a}{q}\right)^{n-m-1} \nabla t+\int_{x}^{b}\left(\frac{b}{q}-t\right)^{n-m-1} \nabla t\right\},
\end{gathered}
$$

$\forall x \in[a, b] \cap q^{\overline{\mathbb{Z}}}$.

Proof. By Theorem 3.5, (3.40).

One can give many similar applications for other time scales. 


\section{References}

[1] G. Anastassiou, Intelligent Mathematics: Computational Analysis, Springer, Heidelberg, New York, 2011.

[2] G. Anastassiou, Nabla Time scales inequalities, Int. J. Dyn. Syst. Differ. Equ. 3 (2011), no. 1-2, 59-83.

[3] G. Anastassiou, Time Scales Delta Iyengar type Inequalities, International Journal of Difference Equations, accepted for publication, 2019, http://campus.mst.edu/ijde.

[4] D. R. Anderson, Taylor Polynomials for nabla dynamic equations on times scales, Panamer. Math. J., 12(4): 17-27, 2002.

[5] D. Anderson, J. Bullock, L. Erbe, A. Peterson, H. Tran, Nabla Dynamic equations on time scales, Panamer. Math. J., 13 (2003), no. 1, 1-47.

[6] F. Atici, D. Biles, A. Lebedinsky, An application of time scales to economics, Mathematical and Computer Modelling, 43 (2006), 718-726.

[7] M. Bohner and A. Peterson, Dynamic equations on time scales: An Introduction with Applications, Birkhaüser, Boston (2001).

[8] S. Hilger, Ein Maßkettenkalkül mit Anwendung auf Zentrumsmannigfaltigkeiten, $\mathrm{PhD}$. thesis, Universität Würzburg, Germany (1988).

[9] K.S.K. Iyengar, Note on an inequality, Math. Student, 6 (1938), 75-76.

[10] N. Martins, D. Torres, Calculus of variations on time scales with nabla derivatives, Nonlinear Analysis, 71, no. 12 (2009), 763-773. 